\title{
Compensatory turning strategies while walking in patients with hip osteoarthritis.
}

\section{$\operatorname{AUTHOR}(S)$ :}

Tateuchi, Hiroshige; Tsukagoshi, Rui; Fukumoto, Yoshihiro; Akiyama, Haruhiko; So, Kazutaka; Kuroda, Yutaka; Ichihashi, Noriaki

\section{CITATION:}

Tateuchi, Hiroshige ... [et al]. Compensatory turning strategies while walking in patients with hip osteoarthritis.. Gait \& posture 2014, 39(4): 1133-1137

\section{ISSUE DATE:}

2014-04

URL:

http://hdl.handle.net/2433/187806

\section{RIGHT:}

(C) 2014 Elsevier B.V.; This is not the published version. Please cite only the published version.; この論文は出版社版でありません。引用の際に は出版社版をご確認ご利用ください。 


\section{Title:}

Compensatory turning strategies while walking in patients with hip osteoarthritis

\section{The name(s) of the author(s):}

Hiroshige Tateuchi ${ }^{\mathrm{a} * *}$, Rui Tsukagoshi ${ }^{\mathrm{b}}$, Yoshihiro Fukumoto ${ }^{\mathrm{c}}$, Haruhiko Akiyama ${ }^{\mathrm{d}}$, Kazutaka So ${ }^{\mathrm{e}}$, Yutaka Kuroda $^{\mathrm{e}}$, Noriaki Ichihashi ${ }^{\mathrm{a}}$

The affiliation(s) of the author(s):

${ }^{a}$ Human Health Sciences, Graduate School of Medicine, Kyoto University, Kyoto, Japan

${ }^{\mathrm{b}}$ School of Rehabilitation, Hyogo University of Health Sciences, Kobe, Japan

${ }^{c}$ The Faculty of Rehabilitation, Kobe Gakuin University, Kobe, Japan

${ }^{\mathrm{d}}$ Department of Orthopaedic Surgery, Gifu University, Gifu, Japan

${ }^{\mathrm{d}}$ Department of Orthopaedic Surgery, Kyoto University, Kyoto, Japan 


\begin{abstract}
The ability to change directions while walking is an integral component of adaptive locomotor behavior. Patients with hip osteoarthritis (OA) experience prolonged hip dysfunction. Gait compensation adopted by the patients with hip OA may become more pronounced while they turn. The purposes of this study were to identify the turning strategy while walking in patients with hip $\mathrm{OA}$, and to examine the relationship between the turning strategy and the patient's functional level. Fourteen patients with hip OA and 13 age-matched healthy controls were recruited. The hip, knee, and ankle joint angles and moments, and the foot progression angle were measured under three walking conditions (straight walking, $45^{\circ}$ step turn, and $45^{\circ}$ crossover turn), and the gait variables for each walking condition were compared between the 2 groups. The relationship between the increasing rate of knee and ankle joint moments in the turning to the straight walking and the functional point in the Harris hip score (HHS) was examined. The OA group showed decreased hip flexion, extension, and abduction angles, and hip flexion moment during the step turn, and decreased hip flexion, extension, and adduction angles, and hip abduction moment during the crossover turn. Furthermore, the ankle plantarflexion moment and the change in the foot angle during the stance phase were significantly increased during the crossover turn in the OA group. The increasing rate of the ankle plantarflexion moment correlated significantly with the functional point in the HHS. Patients with hip OA rely primarily on the ankle plantarflexors to compensate for the hip dysfunction while changing the walking direction.
\end{abstract}




\section{Introduction}

Patients with hip osteoarthritis (OA) experience prolonged pain, limited joint range of motion, and muscle weakness. Previously conducted studies have analyzed the kinematic and kinetic adaptations during walking in patients with hip OA and compared them with those in healthy individuals; the patients commonly show decreased walking speed, step and stride length, hip joint motion, and hip muscle moment and power [1-5]. However, all of these findings were associated with gait characteristics in patients with hip OA during straight walking.

Changing direction of travel while walking is essential for functional mobility and is a common occurrence in activities of daily living [6]. It is an integral component of adaptive locomotor behavior. When changing direction, the body's center of mass must be controlled in the mediolateral plane and the body has to be reoriented to the direction of the intended travel [7]. Commonly, the step turn and crossover turn (also known as spin turn) are used for changing direction while walking [8,9]. As for the hip joint, in comparison to straight walking, the step turn required increased hip abduction angle and the crossover turn required increased hip abduction and internal rotation moments especially during late stance $[10,11]$. Therefore, the underlying movement strategy of the patients with hip OA, which is not apparent during straight walking, may become more pronounced while they turn, and an analysis of this turning strategy would provide clarification regarding the strategy used by the patients during daily activities. However, to the best of our knowledge, no studies have examined the strategy used by patients with hip OA while turning. Therefore, the primary purpose of this study was to identify the movement strategy of patients with hip OA during locomotion by examining the strategy adopted while turning and straight walking. Furthermore, it is unclear whether the movement pattern used by patients contributes to maintenance of their functional activity level. Therefore, a secondary purpose of this study was to examine the relationship between the turning strategy while walking and the functional score.

\section{Methods}

\subsection{Patients}

Fourteen female patients with hip OA (OA group: age, $59.3 \pm 5.3$ years; body weight, $53.3 \pm 9.1$ $\mathrm{kg}$; height, $153.3 \pm 5.5 \mathrm{~cm}$; mean $\pm \mathrm{SD}$ ) and 13 female age-matched controls (control group: age, $62.6 \pm 4.4$ years; body weight, $50.6 \pm 5.3 \mathrm{~kg}$; height, $152.7 \pm 4.9 \mathrm{~cm}$ ) were recruited for this study. The mean Harris hip score (HHS; total 100 points, < 70 poor) of the OA group was $61.1 \pm 10.5$ points. All the patients had end-stage hip OA and were scheduled total hip arthroplasty within 1 month at the time of recruitment. Patients were excluded from the study if they had a musculoskeletal condition other than hip OA or if they had been diagnosed with neurological disorders. Patients with a leg length discrepancy greater than $20 \mathrm{~mm}$ were also excluded, as it can 
impair the patient's walking ability significantly [12]. All of the control participants were free from orthopedic and neurological abnormalities. All the participants provided informed consent, and the protocol was approved by the local ethics committee.

\subsection{Testing protocol}

The testing protocol consisted of straight barefoot walking and turning while walking. During the straight walking trials, the participants were asked to walk straight along a 7-m travel path. During the turning trials, the participants were asked to walk straight until the halfway point of the travel path and then make a turn of $45^{\circ}$ to the right or left (Fig. 1). Previous studies have employed turning angles from $20^{\circ}$ to $90^{\circ}$ to identify the turning strategy [7-11, 13-18]; we selected a turning angle of $45^{\circ}$ to ensure that the task was of moderate intensity, thereby minimizing the physical load on the patients. For the OA group, the affected side was in contact with the force plate at the initiation of the turn, and the patients were asked to step with their unaffected side toward the outside (step turn) or the inside across the affected side (crossover turn). The non-dominant side was in contact with the force plate at the initiation of the turn for the control group. The direction of travel was indicated by a colored tape placed on the floor; however, the foot progression angle, while grounded on the force plate, was self-selected. The OA group was asked to walk at a self-selected speed. The control group was asked to walk at a self-selected speed and at an adjusted slow speed to compensate for the expected differences in the walking speed between the 2 groups. Participants were given several practice trials. The start position was adjusted so that the participants could step on the force plate appropriately. The trials were deleted if the foot was contacted out of the force plate or if the opposite leg was in contact with the force plate. The experimental conditions of the walking and turning trials were presented in random order, and 3 successful trials were recorded for subsequent analysis.

\subsection{Gait analysis}

The kinematic and kinetic measurements were recorded using a 7-camera Vicon motion system (Vicon Nexus; Vicon Motion Systems Ltd. Oxford, England) at a sampling rate of $200 \mathrm{~Hz}$ and a 4th order Butterworth low-pass filter with a $6 \mathrm{~Hz}$ cutoff, and using force plates (Kistler Japan Co., Ltd. Tokyo, Japan) at a sampling rate of $1000 \mathrm{~Hz}$ and a low-pass filter (20 Hz). Reflective markers were attached to the body according to the Vicon Plug-in-Gait marker placement protocol (lower body) by a single investigator. Sixteen markers were placed bilaterally, on the anterior superior iliac spine, posterior superior iliac spine, lateral thigh, lateral femoral epicondyle, lateral shank, lateral malleolus, second metatarsal head, and calcaneus.

The Vicon clinical manager software was used to calculate the following gait variables: walking speed, peak joint angle, and peak internal joint moment at the hip joint in the sagittal and frontal planes, and at the knee and ankle joints in the sagittal plane during the stance phase. For the ankle plantarflexion moment, each peak value for the first and the second halves of the stance phase was 
calculated. The joint moments were normalized by body weight and leg length. Furthermore, the foot progression angle, which is the angle between the sagittal laboratory axis and foot longitudinal axis (joining the center of the ankle joint and marker of the second metatarsal head), was measured during the stance phase. For the foot progression angle, the mean angle and the amount of change in the angle during the stance phase were calculated. We analyzed these gait variables for the affected side of the participants in the OA group and for the non-dominant side of the participants in the control group. The mean values from the 3 trials for each of the 3 sessions were used for analysis.

\subsection{Statistical analysis}

The SPSS version 19.0 (IBM Japan Ltd.) was used for statistical analysis. The Shapiro-Wilk test was used to determine whether datasets followed a normal distribution. According to the results of the normality test, the unpaired $t$ test or Mann-Whitney's $U$ test was used to determine whether there were significant differences in the gait variables between the 2 groups for each walking condition. If a significant difference was noted in the gait speed between the 2 groups in each walking condition, we chose the trials with a slow speed for the controls, in the order of the gait speed from the fastest participant, for a comparison of the gait variables between the 2 groups using the speed-matched trials. The gait variables were compared when the difference in the walking speed was no longer found to be significant. Furthermore, when significant difference was identified in the knee or ankle joint moments during turning in the OA group in comparison to the control group, the relationship between the rate of change of the knee or ankle joint moments during turning compared with that during straight walking and the functional point in the HHS (gait and ADL; max, 47 points) was examined using the Spearman rank-correlation coefficient. Statistical differences were defined as significant at the $\alpha=0.05$ level.

\section{Results}

\subsection{Comparison between the $\mathrm{OA}$ and control groups}

Table 1 shows the temporal, kinematic, and kinetic variables of both groups for the 3 walking conditions. The OA group walked at significantly slower speeds compared with the control group during the straight walk, although there was no significant difference in the walking speed between the groups during the step and crossover turns. When walking at a slow speed was adopted for the 2 fastest individuals and analyzed, no significant difference was found between the walking speeds of the 2 groups. Consequently, we were able to compare the kinematic and kinetic data between the 2 groups.

During straight walking, the OA group showed significantly decreased hip flexion, hip extension, hip adduction, and knee flexion angles, and significantly smaller hip flexion, hip extension, and knee flexion moments compared with the control group. There were no significant differences in the 
angles and moments of the ankle joint between the 2 groups.

During the step turn, the hip flexion, extension, and abduction angles, and the hip flexion moment and knee flexion moment were decreased significantly in the OA group compared with the control group. Though not statistically significant, it should be noted that the ankle plantarflexion moment during the first half of the stance phase tended to increase in the OA group.

During the crossover turn, the patients had decreased hip flexion, hip extension, hip adduction, and knee flexion angles, and decreased hip abduction moment. The knee extension moment in late stance was significantly increased in the OA group; however, the values were relatively small through the walking and turning. Meanwhile, the ankle plantarflexion moment during the first half of the stance phase was significantly increased by approximately $25 \%$ in the OA group compared with the control group (Fig. 2).

The OA group showed significantly greater foot progression angle with more toe-out foot placement during straight walking and the crossover turn. The amount of change in the foot progression angle during the stance phase was also significantly increased in the OA group during the crossover turn.

\subsection{Relationship between the compensatory increase of knee or ankle joint moment and the functional point in the Harris hip score}

The OA group showed a significant increase in the knee extension moment in late stance and ankle plantarflexion moment in the first half of the stance phase during the crossover turn, although there was no increase in knee and ankle moments during the step turn. Therefore, correlation coefficients were subsequently computed to examine the relationship between the increasing rate of the knee and ankle moments in the crossover turn compared with that during the straight walking and functional point in the HHS. Although there was no significant relationship between the increasing rate of the knee moment and functional point in the HHS (Fig. 3, $\rho=0.16, p=0.60$ ), the increasing rate of the ankle plantarflexion moment in the first half of the stance phase was significantly correlated with the functional point in the HHS (Fig. 3, $\rho=0.54, p<0.05$ ); that is, functional level was higher in the patients who could generate a greater ankle plantarflexion moment during turning than during straight walking.

\section{Discussion}

The primary finding of this study was that the ankle plantarflexion moment in the first half of the stance phase increased with the increase in amount of change in the foot progression angle during stance phase in the OA group, especially during crossover turning. We also found that the increase in the rate of the ankle plantarflexion moment during the crossover turn compared with that during straight walking was related to a high functional level as evaluated by the HHS. 
The results of our straight walking test were consistent with those of previous studies, with respect to decreased hip motion and hip moments in patients with hip OA compared with those in healthy individuals [1-5]. On the other hand, during turning, the OA group showed decreased hip abduction angle in the step turn and hip adduction angle in the crossover turn, as well as decreased hip flexion and extension angle. This indicates that the turning strategy generated by the expansion of the hip motion toward the stepping direction is modified in patients with hip OA. Meanwhile, the OA group also showed a tendency for increased ankle plantarflexion moment during turning. The ankle plantarflexor and hip flexor strategies have been considered to contribute to forward progression of the leg into the swing phase and to stabilization and forward progression of the body in the late stance [19]. It has been demonstrated in simulated and clinical studies that dysfunction of the hip joint (e.g., reduced hip flexor power generation and increased hip joint stiffness) leads to compensatory mechanisms by the ankle plantarflexor and vice versa [20-23]. However, based on the data obtained in this study, the OA group showed greater ankle plantarflexion moment even in the first half of the stance phase, especially during the crossover turn. Since the action of the ankle plantarflexors during the first half of stance phase decelerates the body [24], it is unlikely that an increased ankle plantarflexion moment from the early stance phase in the OA group contributes to progression of the body.

The OA group showed more toe-out foot placement in the crossover turn, and showed toe-in foot placement in the step turn which was comparable with the controls despite the greater toe-out angle during the straight walking. These findings suggest that patients with hip OA adjust their foot position toward the direction of travel before contacting the ground at the initiation of the turn to reduce the physical demand during turning. Furthermore, the OA group showed a large amount of change in the foot angle during the stance phase of the crossover turn in comparison to the control group. It indicates that the patients used the crossover turn in combination with a pivot turn: that is, the body is raised up onto the forefoot, where rotation occurs on the ball of the foot, and pivoted toward the intended direction. The pivot turn enables changing direction with less range of hip motion than the usual crossover turn, whereas the pivot turn required greater ankle plantarflexion moment than the step and crossover turn [10]. Therefore, in this study, increased ankle plantarflexor moment and increased change in the foot progression angle during the stance phase are related to each other. Previously conducted studies have found that, $40 \%$ of healthy young individuals used the pivot turn rather than the crossover turn when changing direction at $90^{\circ}$, although the prevalence of the pivot turn in turning at $45^{\circ}$ has not been investigated [10]. Patients with hip OA would adopt the strategy depending on the ankle plantarflexor not only to progress the body and leg in the late stance but also to change the direction in compensation for the hip dysfunction. The crossover turn requires greater hip joint motion and muscle strength compared with the step turn [10]. Thus, the compensatory strategy would become more pronounced during the crossover turn than during the step turn. This turning strategy of the OA group during walking might be related to the previous finding that there was no difference in the cross-sectional area of the ankle plantarflexors between 
the affected and non-affected sides in patients with hip OA, although hip muscles and thigh muscles were atrophied on the affected side [25].

It is noteworthy that the patients with hip OA who could raise the ankle plantarflexion moment during the crossover turn had a higher functional score in HHS. This suggests that the compensatory strategy of increasing the ankle plantarflexion moment according to the demands of the task is effective against lowering the functional activity level. Patients with hip OA are less physically active compared with healthy individuals [26], and avoidance of activity is a significant risk factor for future functional decline [27]. Therefore, it is important not to lower the activity level despite the hip dysfunction in patients with hip OA. Our findings might provide clinicians with information for selection of the appropriate therapeutic strategy for conservative treatment in patients with hip OA.

It is important to mention the limitations of this study. We did not calculate the joint angles and moments in the transverse plane. However, the joint range of motion, joint moment, and power in the transverse plane were relatively small while turning [11]. In addition, a previously conducted study showed that transtibial amputees rely primarily on sagittal plane hip joint work to turn, whereas non-amputees rely primarily on ankle work in the sagittal plane and hip joint work in the frontal plane [28]. Therefore, the functions of the hip joint in the sagittal and frontal planes and the ankle joint in the sagittal plane would assume a primary role in the turning strategy. Another limitation was that only the turns with landing on the affected side were analyzed for the OA group. However, patients with hip OA could perform turns with landing on the non-affected side as an anticipatory compensation during activities of daily living. Finally, since this was cross-sectional study, we did not determine whether an increased ankle plantarflexion moment during walking contributes to the improvement of the patient's functional level. Further research is necessary to examine the effects of gait modification on functional improvement in patients with hip OA.

We conclude that patients with hip OA rely primarily on the ankle plantarflexors to compensate for the hip dysfunction while changing direction during walking. Increasing the ankle plantarflexion moment during turning is important essentially for the functional level to not be lowered in patients with hip OA. Understanding the turning strategies during walking will provide insight into the physical therapy appropriate for preservation of the hip joint and for improvement of locomotor function in patients with hip OA.

\section{Acknowledgements}

This study was supported by a Grant-in-Aid for Young Scientists (B) from the Ministry of Education, Culture, Sports, Science and Technology.

\section{Conflict of interest statement}

None of the authors have any conflicts of interest associated with this study. 


\section{References}

[1] Murray MP, Gore DR, Clarkson BH. Walking patterns of patients with unilateral hip pain due to osteo-arthritis and avascular necrosis. J Bone Joint Surg 1971; 53-A: 259-74.

[2] Hurwitz DE, Hulet CH, Andriacchi TP, Rosenberg AG, Galante JO. Gait compensations in patients with osteoarthritis of the hip and their relationship to pain and passive hip motion. $\mathrm{J}$ Orthop Res 1997; 15: 629-35.

[3] Watelain E, Dujardin F, Babier F, Dubois D, Allard P. Pelvic and lower limb compensatory actions of subjects in an early stage of hip osteoarthritis. Arch Phys Med Rehabil 2001; 82: 1705-11.

[4] Bejek Z, Paróczai R, Illyés Á, Kiss RM. The influence of walking speed on gait parameters in healthy people and in patients with osteoarthritis. Knee Surg Sports Traumatol Arthrosc 2006; 14: 612-22.

[5] Kubota M, Shimada S, Kobayashi S, Sasaki S, Kitade I, Mtsumura M, Kamei K, Kitano M, Takeno K, Baba H. Quantitative gait analysis of patients with bilateral hip osteoarthritis excluding the influence of walking speed. J Orthop Sci 2007; 12: 451-7.

[6] Glaister BC, Bernatz GC, Klute GK, Orendurff MS. Video task analysis of turning during activities of daily living. Gait Posture 2007; 25:289-94.

[7] Patla AE, Adkin A, Ballard T. Online steering: coordination and control of body center of mass, head and body reorientation. Exp Brain Res 1999; 129: 629-34.

[8] Patla AE, Prentice S, Robinson C, Neufeld J. Visual control of locomotion: strategies for changing direction and for going over obstacles. J Exp Psychol: Hum Percept Perform 1991; 17: 603-34.

[9] Akram SB, Frank JS, Chenouri S. Turning behavior in healthy older adults: Is there a preference for step versus spin turns? Gait Posture 2010; 31: 23-6.

[10] Taylor MJD, Dabnichki P, Strike SC. A three-dimensional biomechanical comparison between turning strategies during the stance phase of walking. Hum Mov Sci 2005; 24: 558-73.

[11]Xu D, Chow JW, Wang YT. Effects of turn angle and pivot foot on lower extremity kinetics during walk and turn actions. J Appl Biomech 2006; 22: 74-9.

[12] Gurney B. Leg length discrepancy. Gait Posture 2002; 15: 195-206.

[13]Fuller JR, Adkin AL, Vallis LA. Strategies used by older adults to change travel direction. Gait Posture 2007; 25: 393-400.

[14]Crenna P, Carpinella I, Rabuffetti M, Calabrese E, Mazzoleni P, Nemni R, Ferrarin M. The association between impaired turning and normal straight walking in Parkinson's disease. Gait Posture 2007; 26: 172-8.

[15]Paquette MR, Fuller JR, Adkin AL, Vallis LA. Age-related modifications in steering behavior: 
effects of base-of-support constraints at the turn point. Exp Brain Res 2008; 190: 1-9.

[16]Glaister BC, Orendurff MS, Schoen JA, Bernatz GC, Klute GK. Ground reaction forces and impulses during a transient turning maneuver. J Biomech 2008; 41: 3090-3.

[17] Strike SC, Taylor MJD. The temporal-spacial and ground reaction impulses of turning gait: Is turning symmetrical? Gait Posture 2009; 29: 597-602.

[18]Hollands KL, van Vliet P, Zietz D, Wing A, Wright C, Hollands MA. Stroke-related differences in axial body segment coordination during preplanned and reactive changes in walking direction. Exp Brain Res 2010; 202: 591-604.

[19]McGibbon CA. Toward a better understanding of gait changes with age and disablement: neuromuscular adaptation. Exerc Sport Sci Rev 2003; 31: 102-8.

[20]Judge JO, Davis RB, Õunpuu S. Step length reductions in advanced age: The role of ankle and hip kinetics. J Gerontol 1996; 51: M303-12.

[21] Komura T, Nagano A. Evaluation of the influence of muscle deactivation on other muscles and joints during gait motion. J Biomech 2004; 37: 425-36.

[22]Goldberg EJ, Neptune RR. Compensatory strategies during normal walking in response to muscle weakness and increased hip joint stiffness. Gait Posture 2007; 25: 360-7.

[23] Tateuchi H, Tsukagoshi R, Fukumoto Y, Oda S, Ichihashi N. Clin Biomech 2011; 26: 598-604.

[24]Allen JL, Neptune RR. Three-dimensional modular control of human walking. J Biomech 2012; 45: 2157-63.

[25]Rasch A, Byström AH, Dalén N, Martinez-Carranza M, Berg HE. Persisting muscle atrophy two years after replacement of the hip. J Bone Joint Surg [Br] 2009; 91-B: 583-8.

[26]Judd DL, Thomas AC, Dayton MR, Stevens-Lapsley JE. Strength and functional deficits in individuals with hip osteoarthritis compared to healthy, older adults. Disabil Rehabil 2013; Epub ahead of print.

[27]Pisters MF, Veenhof C, van Dijk GM, Heymans MW, Twisk JWR, Dekker J. The course of limitations in activities over 5 years in patients with knee and hip osteoarthritis with moderate functional limitations: risk factors for future functional decline. Osteoarthritis Cartilage 2012; 20: 503-10.

[28] Ventura JD, Segal AD, Klute GK, Neptune RR. Compensatory mechanisms of transtibial amputees during circular turning. Gait Posture 2011; 34: 307-12. 
Table 1

Gait variables of the OA group and control group during straight walking, $45^{\circ}$ step turn, and $45^{\circ}$ crossover turn

\begin{tabular}{|c|c|c|c|c|c|c|}
\hline & \multicolumn{2}{|c|}{ Straight walk } & \multicolumn{2}{|c|}{$45^{\circ}$ step turn } & \multicolumn{2}{|c|}{$45^{\circ}$ crossover turn } \\
\hline & OA group & Control group & OA group & Control group & OA group & Control group \\
\hline \multicolumn{7}{|l|}{ Walking speed $(\mathrm{m} / \mathrm{s})$} \\
\hline Self-selected & $0.99 \pm 0.14 \S$ & $1.09 \pm 0.11$ & $0.84 \pm 0.14$ & $0.91 \pm 0.16$ & $0.90 \pm 0.15$ & $0.97 \pm 0.13$ \\
\hline After adjustment* & $0.99 \pm 0.14$ & $1.07 \pm 0.10$ & $0.84 \pm 0.14$ & $0.91 \pm 0.16$ & $0.90 \pm 0.15$ & $0.97 \pm 0.13$ \\
\hline \multicolumn{7}{|l|}{ Joint angle $\left({ }^{\circ}\right)$} \\
\hline Hip flexion & $21.0 \pm 7.3 \S$ & $28.8 \pm 5.3$ & $20.2 \pm 8.0 \S$ & $27.6 \pm 4.8$ & $20.8 \pm 7.5 \S$ & $28.2 \pm 4.7$ \\
\hline Hip extension & $3.5 \pm 9.3 \S$ & $16.2 \pm 4.7$ & $2.2 \pm 9.3 \S$ & $14.8 \pm 5.4$ & $2.4 \pm 9.5 \S$ & $14.2 \pm 5.2$ \\
\hline Hip abduction & $2.5 \pm 3.1$ & $4.4 \pm 2.3$ & $2.4 \pm 3.2 \S$ & $5.2 \pm 2.3$ & $1.8 \pm 3.1$ & $2.8 \pm 2.4$ \\
\hline Hip adduction & $4.9 \pm 2.4 \S$ & $7.8 \pm 2.8$ & $4.1 \pm 1.9$ & $4.3 \pm 2.7$ & $5.7 \pm 2.4 \S$ & $8.9 \pm 2.6$ \\
\hline Knee extension & $-1.9 \pm 3.8$ & $-0.6 \pm 3.8$ & $-0.5 \pm 3.7$ & $0.9 \pm 4.4$ & $2.7 \pm 3.6$ & $1.2 \pm 4.1$ \\
\hline Knee flexion & $52.0 \pm 6.4 \S$ & $56.5 \pm 4.7$ & $50.7 \pm 7.6$ & $53.7 \pm 5.2$ & $51.5 \pm 7.1 \S$ & $57.2 \pm 4.6$ \\
\hline Ankle dorsiflexion & $16.4 \pm 3.1$ & $15.8 \pm 2.4$ & $15.6 \pm 3.9$ & $15.7 \pm 3.4$ & $14.5 \pm 3.8$ & $14.8 \pm 3.4$ \\
\hline Ankle plantarflexion & $18.4 \pm 7.4$ & $16.6 \pm 5.6$ & $16.1 \pm 7.0$ & $13.3 \pm 4.1$ & $22.1 \pm 8.2$ & $21.3 \pm 7.0$ \\
\hline \multicolumn{7}{|l|}{ Internal joint moment $\quad(\mathrm{N} \cdot \mathrm{m} / \mathrm{kg} \cdot \mathrm{m})$} \\
\hline Hip extension & $0.58 \pm 0.21 \S$ & $0.77 \pm 0.22$ & $0.53 \pm 0.16$ & $0.63 \pm 0.16$ & $0.49 \pm 0.19$ & $0.57 \pm 0.13$ \\
\hline Hip flexion & $0.87 \pm 0.42 \S$ & $1.14 \pm 0.18$ & $0.73 \pm 0.44 \S$ & $1.02 \pm 0.16$ & $0.91 \pm 0.44$ & $1.12 \pm 0.17$ \\
\hline Hip abduction & $1.07 \pm 0.25$ & $1.22 \pm 0.24$ & $1.03 \pm 0.23$ & $1.15 \pm 0.15$ & $1.05 \pm 0.21 \S$ & $1.21 \pm 0.20$ \\
\hline Knee extension (early) & $0.35 \pm 0.28$ & $0.35 \pm 0.34$ & $0.35 \pm 0.27$ & $0.34 \pm 0.28$ & $0.27 \pm 0.23$ & $0.24 \pm 0.24$ \\
\hline Knee flexion & $0.07 \pm 0.16 \S$ & $0.27 \pm 0.15$ & $0.12 \pm 0.21 \S$ & $0.36 \pm 0.18$ & $0.08 \pm 0.24$ & $0.24 \pm 0.19$ \\
\hline Knee extension (late) $\dagger$ & $0.35 \pm 0.14$ & $0.27 \pm 0.07$ & $0.27 \pm 0.15$ & $0.21 \pm 0.04$ & $0.42 \pm 0.16 \S$ & $0.30 \pm 0.11$ \\
\hline Ankle dorsiflexion & $0.13 \pm 0.05$ & $0.13 \pm 0.07$ & $0.15 \pm 0.06$ & $0.15 \pm 0.09$ & $0.10 \pm 0.05$ & $0.10 \pm 0.06$ \\
\hline Ankle plantarflexion (1st) & $1.00 \pm 0.24$ & $0.88 \pm 0.24$ & $1.07 \pm 0.23$ & $0.89 \pm 0.27$ & $1.07 \pm 0.23 \S$ & $0.85 \pm 0.28$ \\
\hline Ankle plantarflexion (2nd) & $1.56 \pm 0.19$ & $1.65 \pm 0.17$ & $1.61 \pm 0.16$ & $1.68 \pm 0.21$ & $1.42 \pm 0.15$ & $1.48 \pm 0.14$ \\
\hline Foot progression angle $\left({ }^{\circ}\right) \dagger$ & $6.9 \pm 4.9 \S$ & $2.2 \pm 5.1$ & $-11.1 \pm 7.5$ & $-15.0 \pm 7.8$ & $26.8 \pm 9.0 \S$ & $19.4 \pm 7.6$ \\
\hline Change of foot progression angle $\left({ }^{\circ}\right.$ ) & $10.1 \pm 2.7$ & $9.6 \pm 2.7$ & $8.2 \pm 3.5$ & $7.1 \pm 1.8$ & $23.0 \pm 7.7 \S$ & $16.3 \pm 4.8$ \\
\hline
\end{tabular}

* The walking task at a slow speed was adopted for 2 control participants to compensate for the difference in self-selected walking speed between the 2 groups.

$\dagger$ Comparison by using Mann-Whitney's $U$ test.

$\$$ Positive value indicates foot angle of toe-out placement.

$\S$ Significant difference between the OA and control groups 


\section{Figure Captions}

Fig. 1.

Footprints of participants during straight walking, step turn at a $45^{\circ}$ angle, and crossover turn at a $45^{\circ}$ angle. The affected side of the patients and non-dominant side of the controls was in contact with the force plate at initiation of the turn (black foot).

Fig. 2.

Averaging waveform of the ankle plantarflexion moment in the stance phase during a crossover turn in the control group (dashed line) and the OA group (solid line).

Fig. 3.

Relationship between the increasing rate of the knee extension moment in the late stance (a) and ankle plantarflexion (PF) moment in the first half of the stance phase (b) in crossover turn to straight walking and functional point in HHS. Functional point in the HHS consists of gait ability and ADL, and maximum point is 47. 
Straight walk

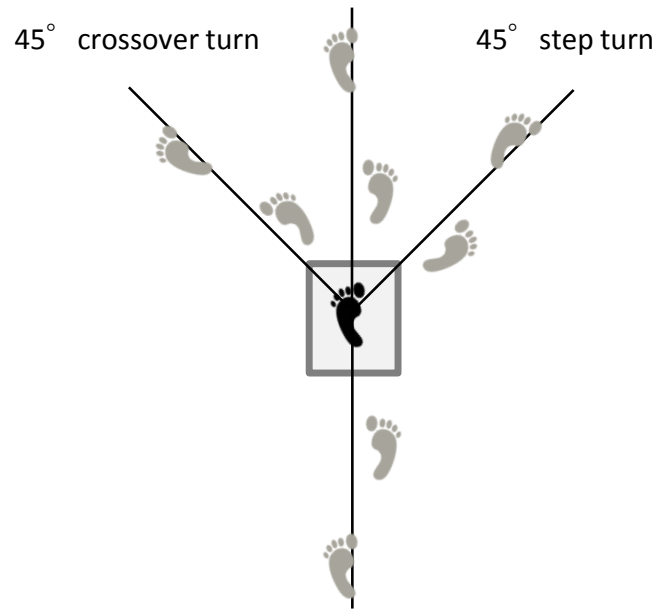

Fig 1. 


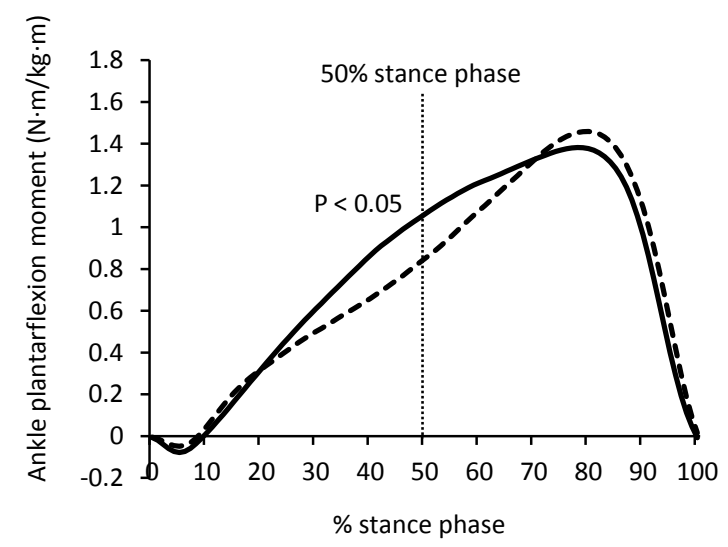

Fig 2. 


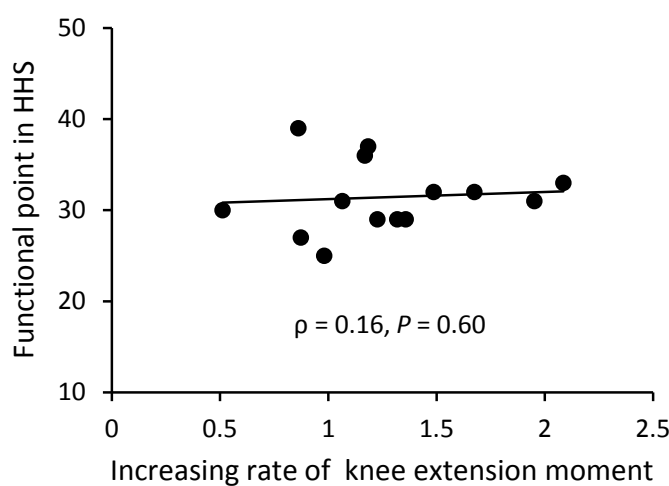

b.

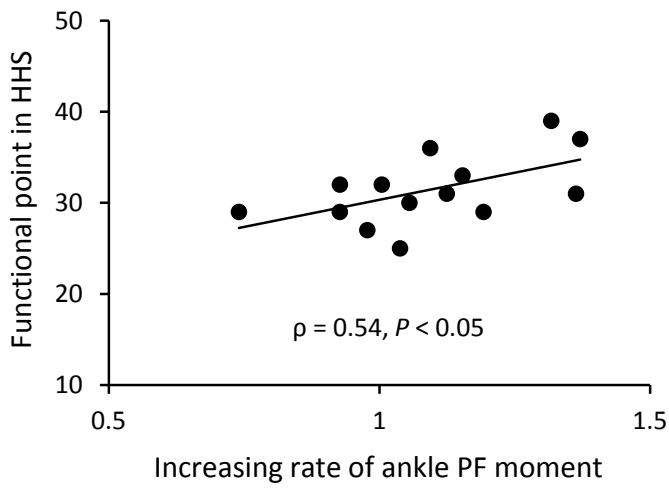

Fig 3. 